\title{
Aprendizaje a lo largo de la vida como estrategia de envejecimiento activo. Caso de estudio de la Universidad de Mayores de Extremadura
} Lifelong Learning as Strategy for Active Ageing. A Case Study of the University of Third Age in Extremadura

\author{
Santiago Cambero Rivero y Deborah Díaz Galván'
}

\section{Resumen}

El aprendizaje es una facultad humana que desarrollamos continuamente mientras aprendemos, incluso antes del propio nacimiento en el útero materno. El aprendizaje como proceso no solo es formal cuando asistimos a centros de enseñanza reglada, sino que también asimilamos mediante las actividades cotidianas. Así, aprendemos a lo largo de la vida, en cualquier etapa del ciclo vital, incluso durante la vejez.

Esta investigación expone la relación teórica y práctica entre el envejecimiento activo y el aprendizaje a lo largo de la vida, como estrategia de conocimiento y adaptación al medio social, y de promoción de la autonomía personal. El trabajo empírico muestra los resultados del estudio cualitativo sobre la experiencia de aprendizaje de adultos mayores, sus actitudes, motivaciones y expectativas en la Universidad de Mayores de Extremadura (UMEX). Un programa de desarrollo científico-cultural de la Universidad de Extremadura, desde hace veinte años, que potencia el proceso de enseñanza-aprendizaje para la conservación y mejora de las cualidades psíquicas y físicas del alumnado sénior en este entorno de educación no formal.

La UMEX, como modelo pedagógico de práctica de aprendizaje a lo largo de la vida, ofrece beneficios cognitivos y relacionales favorables al envejecimiento activo; siendo evidente la importancia de la innovación en el aprendizaje para una vida longeva en comunidades educativas más democráticas, inclusivas y transformadoras en la construcción de sociedades del conocimiento para todas las edades.

\section{Palabras clave}

Aprendizaje a lo largo de la vida, envejecimiento activo, educación en la vejez, personas mayores, universidad, Extremadura.

\section{Abstract}

Learning is a valuable human ability which we are continuously developing while we learn, even before the birthing itself from the maternal womb. Learning as a process is not only a formal way when we attend school regularly, we also learn through daily actions. Thus, we are learning throughout life, at any stage of the life cycle, even during the elderly.

This research exposes the theoretical and factual relationship between active ageing and lifelong learning, as a strategy of knowledge and adaptation to social environment, and the promotion of personal autonomy. The empirical study shows the results of qualitative analysis research on learning experience in older adults, its benefits, expectations, and difficulties, focused on the University of the Third Age of Extremadura. It's an academic programme of scientific-cultural developing offered by the University of Extremadura twenty years ago, with the aim to power of teaching-learning process for the conservation and improvement of the psychic and physical qualities of its senior pupil in this non-formal education environment.

The UMEX, as a pedagogical model of practice of lifelong learning, offers for the cognitive and relational benefits favorable to active aging; so being obvious the importance of innovation in learning for a long life in educational communities more democratic, inclusive and transformative in knowledge societies for all ages.

\section{Keywords}

Lifelong learning, active ageing, educational gerontology, older adults, university, Extremadura. 


\section{Demografía del envejecimiento}

El envejecimiento demográfico es consecuencia de dos factores, primeramente, el incremento de la esperanza de vida al nacimiento, justificada por:

«Estos perfiles demográficos se han producido como producto del desarrollo cientifico, social, económico y politico. Es decir, mejor higiene, mejor alimentación, mejores condiciones sanitarias, mejor educación, más alto nivel económico y menor desigualdad social; todas estas condiciones han contribuido a drásticos cambios en un indicador tan importante como es la esperanza de vida como expresión de la salud de un pais». (Caprara, 2017: 22).

En el año 2000, la esperanza de vida para el hombre se situaba en 75,92 años y en 82,73 para la mujer en nuestro país. Esta expectativa en 2017 alcanzó el 83,1 años -mismos valores que en 2016-, siendo en los hombres los 80,40 años y en las mujeres los 85,70 años (INE, 2017).

Y el segundo factor, la disminución de la natalidad y fecundidad, que se han reducido progresivamente en las últimas décadas en España, como refleja la tasa de natalidad (nacimientos por cada 1.000 habitantes) del 8,37 (ibíd.). El número medio de hijos por mujer se situó en 1,31 en 2017, con un descenso de tres centésimas respecto al valor registrado en 2016, elevándose la edad media a la maternidad a 32,1 años, frente a los 32,0 de 2016 (ibíd.).

En este contexto de madurez demográfica (Pérez Díaz, 2003), la población española envejece y se estima que seguirá sucesivamente, mientras los baby boomers-nacidos entre 1945 y 1960 en España- alcancen la edad de jubilación. Según datos del Padrón Continuo (INE), a 1 de enero de 2017, hay 8764204 personas mayores (65 y más años), un 18,8\% sobre el total de la población (46.572.132) (Abellán et al., 2018). Según proyecciones demográficas de distintos organismos, España podría convertirse en uno de los países más envejecidos del mundo rozando el 40\% de población mayor de 60 años en 2040 (ECC, 2017).

Ante esta revolución demográfica, debemos estar preparados desde cualquier ámbito de la sociedad, atendiendo multidisciplinariamente el envejecimiento y la vejez. Cada día se alcanzan edades más avanzadas - ser centenario no resulta una anécdota, sino una realidad social en España-, de modo que afrontaremos estados de vejez más extensos temporalmente, como preparación para el envejecimiento activo y saludable.

«El envejecimiento es un proceso lento, en el que trascurre ya más de un tercio de nuestra vida y durante el que se debe garantizar a las personas mayores el derecho a gozar de oportunidades de formación y empleo, el de participación activa en la vida familiary social a través de actividades de voluntariado, el aprendizaje permanente, la expresión cultural y el deporte». (Martínez, 2011: 19).

\section{Aprendizaje a lo largo de la vida}

El objeto de estudio es el aprendizaje a lo largo de la vida (ALV) como estrategia de envejecimiento activo (EA), focalizado en el estudio de caso del programa universitario «Universidad de Mayores de Extremadura» (UMEX). El ALV como práctica de enseñanza-aprendizaje durante el ciclo vital, es conocido con el término inglés «lifelong learning», definido por la Comisión Europea como:

«El aprendizaje a lo largo de la vida es el desarrollo del potencial humano a través de un proceso sustentador continuo que estimula y faculta a los individuos para adquirir todos los conocimientos, valores, destrezas y comprensión que requieran a lo largo de toda su vida y aplicarlos con confianza, creatividad y gozo en todos los roles, circunstancias y entornos». (Comisión Europea, 1995). 
Las múltiples definiciones del ALV comparten elementos sobre su carácter longitudinal en el sentido temporal hasta el final de la vida, su carácter vertical abarcando todas las dimensiones personales, la complementariedad de oportunidades de enseñanza formal, no formal e informal, y la flexibilidad y diversidad en los contenidos y técnicas de aprendizaje conectados con las experiencias educativas previas del individuo (IMSERSO, 2011).

En el año 2014, la UNESCO marcó su estrategia de educación 2014-2021, que señalaba como uno de los objetivos estratégicos para la consecución del desarrollo sostenible, el progreso y la igualdad. «Desarrollar sistemas de educación que fomenten un aprendizaje de calidad e inclusivo a lo largo de toda la vida para todos». (UNESCO, 2014: 37).

Con anterioridad, el informe denominado Jacques Delors (1996), publicado por encargo de la UNESCO, con el título La educación encierra un tesoro, estimuló el diseño de políticas educativas a nivel mundial, y en la actualidad sigue conceptualizando el ALV. En el mismo, se subraya que «estar en condiciones de aprovechar y utilizar durante toda la vida cada oportunidad que se le presente de actualizar, profundizar, y enriquecer ese primer saber y de adaptarse a un mundo en permanente cambio». (Delors, 1996: 95).

En el año 2015, se publica el Plan Estratégico de Aprendizaje a lo Largo de la Vida en España por el Ministerio de Educación, Cultura y Deporte, estableciendo objetivos para su implementación por las administraciones educativas:

«1. Generalizar el acceso a la información, orientación y asesoramiento para que todos los ciudadanos puedan participar en la formación permanente.

2. Mejorar la calidad de la formación permanente.

3. Fomentar la innovación en educación permanente.

4. Adaptar la oferta formativa a las necesidades personales, sociales y laborales de los ciudadanos.

5. Flexibilizar y conectar los sistemas e itinerarios formativos.

6. Incrementar el porcentaje de ciudadanos que participan en actividades formativas de formación permanente, asi como los niveles de cualificación de estos.

7. Propiciar la permanencia efectiva de los ciudadanos en las distintas modalidades de formación». (Ministerio de Educación, Cultura y Deporte, 2015: 31).

El ALV gana importancia gradual en sociedades, como la española, dado los indicadores de envejecimiento demográfico. La coyuntura gerontológica ofrece una nueva visión del envejecimiento relacionado con el paradigma del envejecimiento activo, la calidad de vida y las dimensiones de capacidad personal (IMSERSO, 2011). La curiosidad intelectual (orden instrumental) y el aprender por el placer de aprender (orden expresivo) serían las principales motivación de los adultos mayores (Villar y Serrat, 2014), que cubren necesidades de supervivencia frente al envejecimiento, contribución altruista comunitaria, y de influencia y generatividad (Erikson, 1982).

A pesar del impulso institucional del ALV a escala internacional y nacional, la ciudadanía sénior parece no valorar las oportunidades de autorrealización e integración social desde los espacios de formación continua en España. Los datos relativos a los tipos de aprendizaje señalan que son escasas la proporción de personas que optan por el aprendizaje formal a partir de los 45 años, tan sólo un 0,5\% de la población 
comprendida entre 45 y 54 años y un $0,4 \%$ de la población entre 55 y 65 años, frente al 11,5\% y el 8,4\% respectivamente que deciden decantarse por aprendizajes no formales (INE, 2016).

Aunque esta investigación no analiza las causas de la realidad socio-educativa caracterizada por la estadística anterior, si detecta el déficit educativo en el segmento de población adulta mayor en nuestro país, señalando el relativo desinterés entre el alumnado potencial sobre la oferta de espacios educativos, como sería el caso de la UMEX.

Subrayar las bolsas de analfabetismo y población sin estudios en la generación presente de mayores de 60 años en España, que vivieron situaciones de escasez dotacional educativa, además del deterioro de las condiciones de vida tras la posguerra civil. Quizás estos factores ambientales y personales incidan en la valoración escasa del ALV entre los baby boomers. De ahí, la importancia de crear espacios de carácter democrático, donde los diferentes grupos sociales puedan ejercer el derecho a la participación, tomando decisiones de mejora no solo de orden interno, sino también en el contexto social donde está inmersa la institución educativa (Roa y Torres, 2014).

\section{Envejecimiento activo}

El envejecimiento activo (EA) es definido por la OMS (2002) como «el proceso por el que se optimizan las oportunidades de bienestar físico, social y mental durante toda la vida, con el objetivo de ampliar la esperanza de vida saludable, la productividad y la calidad de vida en la vejez».

Para promover el envejecimiento activo, hay factores sobre los que podemos influir, y otros que escapan de nuestra influencia, considerando como primordiales la buena alimentación y el ejercicio físico regular. No olvidemos la importancia de la dimensión social y cognitiva, relacionándonos y ejercitando nuestro cerebro para conservar al máximo sus facultades (Fernández-Ballesteros, 2017).

El término «activo» tiene un significado amplio caracterizado como la participación continua en la esfera privada y comunitaria, la mejora o el mantenimiento de las competencias individuales y la búsqueda de un nuevo modelo de sociedad donde envejecer como protagonistas de sus vidas, siendo el aspecto educativo relevante por las oportunidades para el aprendizaje a lo largo del ciclo vital (Bermejo, 2010).

Los beneficios para las personas mayores implicadas en experiencias educativas incluyen no solo el desarrollo de estrategias efectivas de afrontamiento, sino también el interés por nuevas actividades, la integración social y el desarrollo de nuevas redes sociales; en otras palabras, el aumento de la calidad de vida (Villar y Serrat, 2014), demostrando que la práctica del ALV es una estrategia eficaz para el EA.

Por consiguiente, se pretende integrar a las personas mayores en «una sociedad para todas las edades» (Naciones Unidas, 2000), en la que cada persona, con sus propios derechos y responsabilidades, tenga una función activa que desempeñar en sociedades integradoras y respetuosas con los derechos humanos. Así, esta investigación focaliza en la comunidad educativa de la UMEX, como espacio para el ejercicio continuo del derecho a la educación de las personas mayores.

\section{Envejecimiento Activo y Aprendizaje a lo Largo de la Vida}

El envejecimiento no es cuestión de azar, sino que depende de los comportamientos y las elecciones del individuo a lo largo del ciclo vital completo (Fernández-Ballesteros, 2000). La OMS (2002) fijó una serie de factores poblacionales para la consecución del envejecimiento activo (EA), que se destacan a continuación. 
Primeramente, los determinantes conductuales, como la adopción de estilos de vida saludables, la actividad física, la buena alimentación, etc., son decisiones individuales que afectarían al proceso de envejecimiento. A través de la enseñanza se promueven hábitos de vida saludable, tanto para niños y jóvenes, como para adultos mayores, a través de la educación de adultos o de las universidades y aulas de mayores.

Entre los determinantes relacionados con los factores personales, inciden algunos como la biología o la genética sobre los que es meramente difícil influir, y otros como los factores psicológicos (inteligencia, resolución de problemas, adaptación al cambio, motivación,...) sobre las que el ALV si puede depender. Aunque con la edad algunas capacidades cognitivas disminuyen, estas carencias se compensan, e incluso disminuyen, si mantenemos activa la capacidad de conocimiento, las expectativas de aprendizaje, y nos beneficiamos de la confianza y seguridad que aporta cada enseñanza.

Los determinantes concernientes al entorno también guardan relación con el aprendizaje. Primeramente, los entornos para el aprendizaje previenen que los adultos mayores sufran soledad o aislamiento, creando redes de interacción social. Y en segundo lugar, el ALV ayuda a la adquisición de aptitudes y actitudes de adaptación al cambio, desde el empoderamiento y la promoción de la autonomía personal según envejecemos.

\section{«La oportunidad de adquirir nuevos o reciclar conocimientos resulta una experiencia personal estra- tégica para aquellas personas mayores que optimicen sus condiciones intelectuales, estado de salud, autoestima y autonomía, e integración social, entre otros beneficios para la mejora de la calidad de vida durante la vejez’." (Cambero, 2015:165).}

Por consiguiente, como indica el Libro Blanco sobre Envejecimiento Activo (2011), el ALV se dirige hacia el desarrollo de las competencias para la vida, con capacidades de autoconocimiento y autovaloración, creatividad personal, participación en contextos sociales o adquisición de conocimientos culturales y científicos, entre otras competencias individuales (2011: 290).

De modo que el EA expresa elementos del ALV, cuando envejecer supone afrontar cambios personales y adaptarse a una sociedad donde se producen avances acelerados que caducan la validez de la formación anterior. Los adultos necesitan disponer de posibilidades de aprendizaje para el logro de sus exigencias individuales y colectivas, mediante el intercambio de significados, conocimientos, estrategias o experiencias para desarrollar sus habilidades y saberes en la mejora de la vida (IMSERSO, 2011).

Los cambios sociales nos situarán en un nuevo escenario en el que interesarnos por la formación actualizada bajo parámetros de la sociedad de la información y del conocimiento, basada en competencias digitales, herramientas tecnológicas, nuevos códigos lingüísticos, entornos virtuales de aprendizaje, etc. En ese contexto emergente, los adultos mayores querrán desarrollar habilidades y capacidades que hasta el momento no habían descubierto debido a las condiciones de vida pasadas (Ibídem).

\section{Origen de universidades para mayores}

Dado que esta investigación focaliza en la UMEX, conviene conocer ciertas reseñas sobre la evolución histórica de los programas universitarios hasta la actualidad en Europa.

La primera experiencia fue la Université du Troisième Age en 1973, impulsada por el profesor Pierre Vellas desde la Facultad de Ciencias Sociales en Tolouse (Francia). El principal objetivo sería la promoción entre los adultos mayores de actividades académicas que respetaran sus condiciones, necesidades y aspi- 
raciones. Al principio, estas instituciones trataban únicamente de acoger a los adultos mayores, de ahí el término «troisième âge» (Vellas, 1977). Iniciativas análogas se desarrollaron rápidamente en Francia, como por ejemplo, en 1975 en Strasbourg, en 1976 en Rennes, en 1977 en Orléans, en 1978 en Annecy.

Durante los noventa en Francia, se valoró la conveniencia de atender a grupos más amplios de personas que tuvieran tiempo libre, sin imponer condición alguna en cuanto a diplomas, nivel de estudios o profesión, o franjas etarias. Por esta ampliación de la población-meta, se derivaron una serie de denominaciones para estas instituciones, como fueron la universidad de tiempo disponible, o de tiempo libre, o para toda edad, o del tercer tiempo, etc. (Chamahian, 2005).

Tras estas primeras experiencias de entornos de aprendizaje para adultos mayores en Francia, se extendieron igualmente al resto de Europa, surgiendo otros modelos con similares fines socio-educativos. En España, los programas universitarios para mayores se iniciaron en Cataluña, en los años 80. En 1999 existían 20 programas universitarios con sus respectivas delegaciones (Velázquez y Fernández, 1999: 36). A nivel estatal, existe una asociación que acoge las universidades que prestan formación a mayores como es AEPUM (Asociación Estatal de Programas Universitarios para Mayores), fundada en 2004, y acogiendo a 45 universidades españolas actualmente.

Como muestra la siguiente gráfica, no solo ha crecido el número de universidades asociadas, sino también el número de matriculaciones de alumnado sénior.

\section{Gráfico 1. Evolución del número de Universidades y alumnado total matriculado}

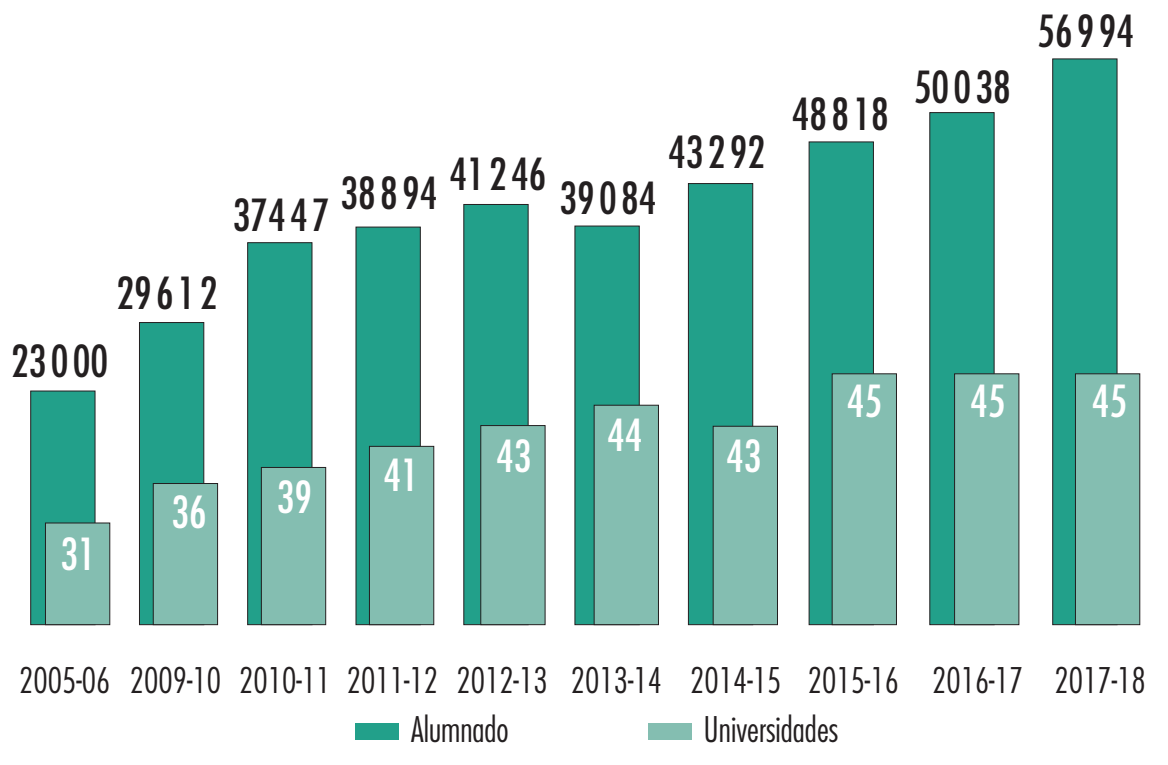

Fuente: AEPUM, 2017

Aunque cada universidad tiene sus propios objetivos, basándonos en las aportaciones de Montero (2000), se enumeran los siguientes:

1. Posibilitar el acceso a las personas mayores a ofertas formativas.

2. Propiciar la participación social de la persona mayor a través de diversas actividades.

3. Contribuir a una mayor realización personal mediante el reconocimiento de su bagaje cultural y la propia valoración de sí mismos. 
4. Propiciar el cambio de la imagen de la persona mayor en la sociedad como componente activo de la misma en la dinámica histórico-cultural.

5. Favorecer las relaciones tanto entre las propias personas mayores como con otras generaciones universitarias.

Se debería añadir la alfabetización digital de las personas mayores en la sociedad telemática (Cambero, 2018; Baigorri y Chaves, 2005), dada la necesidad de nuevas habilidades tecnológicas para usar las TIC, que además están generando tecnologías del aprendizaje y el conocimiento (TAC).

En definitiva, los programas universitarios para mayores son herramientas de educación para mayores, que dada la extensión temporal de la vejez, están llamadas a atender a generaciones que no tuvieron oportunidades educativas, además de ser un medio para prevenir y paliar las deficiencias geriátricas, fomentando así un envejecimiento activo, inclusivo y generativo (Villar y Serrat, 2014).

\section{Universidad de Mayores de Extremadura}

La Universidad de Extremadura ofrece a los mayores de 55 años la posibilidad de formarse a través de la UMEX, desde 1998. Este programa universitario promueve la ciencia y la cultura en las personas mayores al mismo tiempo que las relaciones intergeneracionales, facilitando su desarrollo personal y social, con independencia de su localidad de residencia o nivel de renta, sin requerir de titulación académica previa.

Entre los objetivos de la UMEX destacan los siguientes:

- Facilitar el acceso de las personas mayores a los bienes culturales para la mejora de su calidad de vida y el fomento del ocio creativo.

- Propiciar un espacio para el debate científico-cultural a una generación con escasas oportunidades para el mismo.

- Ofrecer un marco para las relaciones intergeneracionales que, en otros contextos, fueron difíciles.

- Incorporar a los mayores a la sociedad de la información, fomentando la participación como dinamizadores en su contexto socio-cultural.

La UMEX cuenta con siete sedes repartidas por la geografía extremeña, donde se imparten clases en horario de tarde dos veces en semana, en el Campus de Badajoz y de Cáceres, Centros Universitarios de Mérida y de Plasencia, Centros Cívicos de Almendralejo, Don Benito y Villanueva de la Serena y en el IES «Suárez de Figueroa» en Zafra.

La UMEX oferta cinco cursos académicos, con ocho asignaturas diferentes por año, entre las que predominan las humanidades. Además del programa de estudios, se complementa con talleres de expresión plástica, educación física o portugués, y otras actividades extra-académicas como viajes de estudios, teatro o cine-fórum. Finalizado el programa académico, se otorga al alumnado su diploma de graduado de la Universidad de Extremadura, que no es válido para el ejercicio profesional. Para el alumnado que desee continuar se ofrecen cursos de postgrado, que cada año proporcionan nuevos contenidos académicos.

El precio de la matrícula ronda los $70-80 €$ anuales, aunque se conceden becas para la misma y ayudas para los desplazamientos de aquellas personas que cumplen los requisitos establecidos por la Comisión de Becas. 


\section{Objetivos de la investigación}

Como se informaba, esta investigación pretende conocer la experiencia educativa del alumnado sénior de la UMEX, complementado con las valoraciones del profesorado como miembros de esta comunidad universitaria. Tal objetivo genérico se desglosa en los siguientes específicos:

1. Conocer las motivaciones, expectativas y beneficios para el alumnado sénior de la UMEX.

2. Conocer las actitudes del alumnado sénior sobre este tipo de programas universitarios para la educación de personas mayores.

3. Conocer las actitudes del profesorado de la UMEX respecto al alumnado sénior.

4. Establecer las relaciones entre el ALV y el EA desde las aportaciones del alumnado y el profesorado de la UMEX

\section{Diseño metodológico}

Esta investigación empírica describe inductivamente la realidad factual de la comunidad educativa de la UMEX, centrada en el alumnado sénior como unidad de observación principal, para comprender las relaciones entre esta práctica de ALV y el EA de estos adultos mayores. También, se conocieron previamente las opiniones de algunos responsables y docentes de este programa universitario, con la finalidad de delimitar el objeto de estudio y las categorías de análisis que describan los procesos y hechos sociales relativos a este alumnado sénior.

Se formula la hipótesis inicial que este alumnado está compuesto por personas con diferentes niveles de acceso informativo y conocimiento para participar en este programa universitario, que facilita el desarrollo personal y social, sea cual sea su renta disponible y localidad de residencia en Extremadura. De ahí, el interés de conocer en profundidad sus motivaciones, expectativas y beneficios de tal experiencia universitaria, además de opiniones sobre aspectos estructurales y funcionales de la UMEX.

En cuanto a la metodología, se utilizaron técnicas de investigación cualitativas como grupos de discusión, entrevistas y observación participante. Estas técnicas se adaptaban a las unidades de observación y al medio de la UMEX, siendo aplicadas tanto al profesorado como al alumnado, aunque estos últimos actuaron como informante-claves. De ahí, surgen las diferentes categorías de análisis cualitativas (actitudes, motivaciones, expectativas, etc.) para proceder a su interpretación y elaboración del discurso sobre la relación entre el ALV y el EA.

En la fase inicial preparatoria, se estableció el marco teórico-conceptual y la planificación de las actividades a ejecutar en fases posteriores. Se consideraron las posibles reacciones del alumnado ante la solicitud de colaboración en esta investigación y la aplicación de las técnicas más adecuadas a las peculiaridades de los adultos mayores.

Primeramente, se organizaron tres entrevistas con profesorado y el coordinador general de la UMEX, utilizando un guión semi-estructurado de cuestiones de interés para profundizar conocimientos sobre el funcionamiento y la organización de la UMEX, que fueron grabadas y anotadas para su trascripción. La primera entrevista fue realizada al coordinador del programa, Florentino Blázquez Entonado, quien relató el origen y evolución del programa, además del grado de satisfacción general y el progreso de matriculaciones durante los últimos veinte años. Esta persona nos autorizó el desarrollo de la investigación empírica y facilitó los contactos del profesorado de la UMEX, de modo que se realizó una primera en- 
trevista a Fernando, profesor de Sociología, y a continuación, por separado a Jacinto y Marisi, profesores de Literatura.

Se planificaron distintos momentos de observación participante, tanto en el medio propiamente académico como relacional de la UMEX (café entre clases, jornadas, excursiones, etc.), durante los meses de abril y mayo de 2018 ( $2^{\circ}$ cuatrimestre del curso 2017/18). Esto supuso una inmersión en las dinámicas de este alumnado sénior, que asistía dos tardes cada semana (martes y jueves, de 17:00 h. a 20:00 h.) a la Facultad de Educación, como sede en el campus de Badajoz.

La observación en el desarrollo de las clases en los días señalados, conforme al programa de estudios -de $1^{\circ}$ a $5^{\circ}$ más Postgrado-, permitió comparar por cada curso seleccionado en cuanto a comportamientos individuales y grupales del alumnado. Esta relación directa facilitó el hecho de conocer quiénes eran el alumnado, el medio de interacción lectiva y no lectiva, el grado de satisfacción y expectativas, las críticas de mejora de la UMEX, y desde luego, la comprensión del fenómeno de este alumnado sénior en la Universidad de Extremadura. Todos estos datos se registraron en cuadernos del observador, pudiendo establecer variables cualitativas (edad, sexo, ocupaciones, estado civil, residencia, etc.) mediante indicadores observables (proporción de alumnado con diferentes niveles académicos, años de jubilación, categorías laborales-profesionales, etc.). Como resultado de este conocimiento generalizado, se fueron seleccionando a adultos mayores que serían los informante-claves para su convocatoria posterior a los grupos de discusión planificados.

Los grupos de discusión se realizaron mediante convocatorias personalizadas del alumnado en días previos en las clases, explicándoles el objeto de estudio y la importancia de sus aportaciones para generar discurso con valor científico-social, que sería divulgado a través de revistas y congresos especializados en Sociología de la Educación y de la Vejez. Se solicitó el consentimiento informado de los participantes, indicando únicamente nombres y edades de los mismos, como se contempla en el análisis e interpretación de resultados.

Se organizaron cuatro grupos de discusión, en los que participaron un total de veintisiete alumnos y alumnas $-63 \%$ hombres y $37 \%$ mujeres de edades comprendidas entre 58 y 81 años-, de cursos de $2^{\circ}, 3^{\circ}$ y Postgrado. En el primer grupo participaron seis alumnas de $2^{\circ}$ curso, mientras que en el segundo fueron nueve alumnos y alumnas de $2^{\circ}$ y $3^{\circ}$ curso, con edades comprendidas entre 59 y 65 años. El tercero fue un grupo de seis alumnos de segundo curso, con edades comprendidas entre 61 y 66 años. Y el cuarto y último grupo estuvo formado por seis alumnos de Postgrado, con edades comprendidas entre 73 y 78 años.

Los grupos de discusión duraban entre 60 y 75 minutos aproximadamente, siendo dinamizados por uno de los investigadores, previa elaboración de un guión semi-estructuado sobre sus motivaciones, expectativas y beneficios de la experiencia universitaria, además de abordar aspectos relacionales y organizativos sobre la UMEX.

\section{Análisis e interpretación de resultados}

Como tareas de la investigación, se transcribieron las conversaciones de las entrevistas y los grupos de discusión registradas por grabación de audio y anotaciones, además de las observaciones realizadas fuera y dentro de clases, para su análisis de datos e interpretación desde la perspectiva comparada de sendos perfiles: alumnado y profesorado de la UMEX. 
El tratamiento de los datos se efectúo preservando su naturaleza textual, siendo seleccionada y simplificada mediante la categorización y codificación de elementos significativos. Las categorías de análisis se fueron definiendo de forma exhaustiva, pertinente y objetiva, a medida que se examinaban inductiva y deductivamente los datos. Con lo cual, la identificación y clasificación de los datos facilitó la creación de conceptos teóricos representativos. Todas estas tareas se implementaron según lo planificado por la experiencia en la aplicación de mencionadas técnicas cualitativas.

A continuación, se relatan las perspectivas del alumnado y el profesorado de la UMEX participantes en los grupos de discusión y las entrevistas, respectivamente.

\subsection{Perspectiva del alumnado}

\subsubsection{UMEX: la gran desconocida}

Según comentarios del alumnado, se evidencia el desconocimiento generalizado sobre la UMEX en la sociedad extremeña, incluso en la misma Universidad de Extremadura, tras 20 años de cursos. Esta falta de información se observa tanto entre la ciudadanía que guarda escasa o nula relación con el medio universitario, como entre el propio alumnado de la UMEX en sus primeros inicios.

«A mi me preguntan mucho qué carrera estoy haciendo...». (Juan Carlos, 65 años, GD2).

«Muchos lo que dicen es que a qué venimos, para qué vamos a aprender esto si no nos sirve para nada...». (Juan, 74 años, GD4).

Se cree que la UMEX es un espacio de formación académico para quienes no tuvieron la oportunidad de acceder a la enseñanza superior durante la juventud, y que ahora pueden como adultos mayores. La realidad es distinta, como comentaron.

"Hay gente que piensa, como yo mismo al principio que esto sería un rollo, desde mi ignorancia. Después fue todo lo contrario». (Juan Carlos, 65 años, GD2).

"Pensé que aqui venía la gente sin carreras y cuando veía a alguno que era médico o abogado, yo pensaba: ¿Por qué vendrán aqui si tienen estudios?». (Ángela, 58 años, GD1).

\subsubsection{Motivaciones}

La mayoría del alumnado conoce la UMEX por otros usuarios, de manera que alguien conoce a otra persona que le cuenta su experiencia (boca a boca) y se sintieron atraídos por participar en este programa universitario. El número de matriculaciones crece cada año por las buenas referencias y se estima que seguirá así, dado que en el curso pasado 2017/18 se matricularon alrededor de 2.150 personas.

«Me bablaron muy bien de ella, compañeros que están en otros cursos, y me gustó la idea». (Manolo, 62 años, GD2).

"Tenia dos compañeras que hablaban de las clases, de las excursiones y de lo bien que se lo pasaban, las relaciones, y dije: oye que bien os lo pasáis mientras adquieres algo más de cultura y conoces a gente...». (Ángela, 58 años, GD1). 
"Lo que nosotros aprendemos aqui lo hablamos con los demás y esa ilusión la transmitimos a los demás, quienes van apareciendo poco a poco...». (Manolo, 62 años, GD2).

Según relataron, los principales motivos de matriculación y asistencia lectiva se resumían en:

- Aprendizaje continuo para mantener la mente activa.

- Aprovechamiento del tiempo libre tras la jubilación.

- Relaciones interpersonales y vínculos de amistad.

"Una vez que te jubilas tienes dos opciones, o te encierras en ti mismo, o te abres y creces, y esto es una forma de seguir creciendo». (Juan, 78 años, GD4).

"Para mi fue seguir un poco dándole actividad a la mente, no tener la cabeza "plana" después de jubilarte, sino mantener la curiosidad». (Rafael, 75 años, GD4).

«... tuve la desgracia de no poder estudiar de joven, y pensé que sería una buena oportunidad para aprender». (J. Antonio, 63 años, GD3).

«... quería ampliar los conocimientos, tener nuevas amistades...». (J. Enrique, 65 años, GD3).

«Me apunté para aprender y para que la mente fuera trabajando, y asi retrasar al máximo posible la enfermedad, si es que viene alguna de tipo Alsheimer». (Ángeles, 72 años, GD1).

\subsubsection{Expectativas y beneficios}

Por un lado, las expectativas del alumnado, y por otro, los beneficios particulares al asistir a clases. Las expectativas suelen quedar satisfechas, incluso superadas en muchas ocasiones por esta primera experiencia universitaria. Hay personas que reconocen que la UMEX les cambió sus vidas en la vejez.

"Es seguir aprendiendo en la vida, mantenerme activa y viva sin echar cuenta a la edad...». (María José, 74 años, GD1).

«Es como un regalo de la sociedad a las personas que han trabajado tanto». (Juan, 74 años, GD4).

«... me estoy descubriendo mucho más, estoy descubriendo facetas que desconocía gracias a la universidad...». (Teresa, 65 años, GD1).

Algunos de estos adultos mayores acuden para dedicarse tiempo a sí mismos y sentir cierta utilidad social, más allá de sus contribuciones familiares.

«La vida te la llena mucho la familia, pero no todo son los nietos; esto es un complemento bueno para cultivarte por dentro, pues a veces hay que 'desnietarse'». (Juan, 78 años, GD4).

Uno de los puntos fuertes son las relaciones interpersonales alrededor de la UMEX. Sería la acción social de la Universidad de Extremadura mediante la implementación de este programa singular por su alumnado, que palia los efectos de la soledad no deseada y la exclusión del mayor. Los vínculos de amistad creados compensan las carencias relacionales que los sujetos puedan presentar en sus entor- 
nos familiares. De hecho, estas «nuevas amistades universitarias» traspasan el espacio de la UMEX para organizarse reuniones o encuentros para comer, ir al cine, viajar, etc.

Observamos que los periodos de descanso entre clases resultan momentos para conocerse, conversar, compartir ideas y sentirse miembro de esta comunidad universitaria, en un ambiente de igualdad como cohorte de edad.

"Las clases se paran para que exista ese contacto (...), todos mis amigos son de la universidad, o parten de abi, aunque después se amplien». (María José, 74 años, GD1).

"Nosotros que nos hemos conocido aquí, nos llamamos "El puchero" y una vez al mes nos juntamos para comer en diferentes sitios..."». (Fernando, 61 años, GD3).

"Clotilde, no tiene aqui a su bija, está en Suiza; mi bija una está en Madrid y nosotras estamos juntas». (Ángeles, 71 años, GD1).

\subsubsection{EA}

Estos universitarios sénior manifestaron que la UMEX es una fuente de conocimientos y relaciones interpersonales en edades avanzadas. De ahí, que este programa universitario esté promoviendo el EA a través de la práctica del ALV, dado que las personas necesitamos estar en formación continua para mantener conocimientos actualizados en materia de salud, economía, medio ambiente, etc.

«Ampliar la cultura, que es interminable, nunca se acaba de aprender.». (J. Carlos, 65 años, GD2).

«... destaco neuropsicología, una materia con la que hemos descubierto mucho y en una edad en la que replantearnos que tenemos un cuerpo que envejece y que cuidar». (Francisco, 65 años, GD3).

"Todo tiene su utilidad, hay materias que son muy buenas y te ayudan a entender el porqué de muchos fenómenos». (Emilio, 78 años, GD4).

El aprender contenidos académico-científicos no es realmente significativo, pues lo importante es lo que acompaña a ese hecho que aporta al desarrollo personal. Estos adultos mayores establecen relaciones sociales con sus iguales, propiciando que sean personas con conductas prosociales que contribuyan al bienestar general, como evidencian ciertas iniciativas solidarias a lo largo del curso.

Este alumnado está aprendiendo o actualizando sus conocimientos tras la jubilación, sin un sentido utilitarista, sino para mejorar la autoestima y la autoconfianza cuando se aprende, sabe, descubre,..., aquello que desconocía y siente que prospera en su vida. En la UMEX no cabe la apatía, ni la desilusión, pues la componen personas dispuestas a seguir participando activamente en la transformación de la sociedad.

«A veces lo pienso, ¿nosotros qué hacemos ya jubilados?». (Maribel, 65 años, GD2).

"La calidad de vida ha mejorado gracias a la actividad que supone venir a la universidad, sino te quedas en casa, te aíslas». (Rafael, 75 años, GD4). 
«Tienes que buscar algo, porque el cuerpo te está pidiendo moverte y seguir la dinámica de la actividad que twiste durante tantos años de trabajo». (Manolo, 62 años, GD2).

«Ilusión, cultura y amistad, así resumo la UMEX». (Teresa, 65 años, GD1).

Por otra parte, la UMEX también promueve la actividad física-deportiva, pues hay talleres sobre educación física con bastante aceptación entre el alumnado. Además, la acción de desplazamiento para asistir a clases y participar en otras actividades, tales como viajes culturales o caminatas senderistas, favorecen el bienestar físico.

\subsubsection{ALV}

Las clases se imparten mediante charlas-coloquios por el profesorado, conforme al programa de estudios de cada curso. Estar exentos de exámenes o pruebas de conocimiento tranquiliza al alumnado para afrontar cada aprendizaje. El alumnado carece de miedo a las clases, disfruta de ellas, pues no hay presión alguna, lo que favorece que el aprendizaje sea automático cuando se atiende a los contenidos.

«Antes era más obligación, y ahora es más devoción. A esto vienes porque quieres». (J. Carlos, 65 años, GD2).

«Por eso la ilusión ahora es más alta, porque vienes porque te gusta y no te exigen» (Manolo, 62 años, GD2).

«Aprender abora me resulta más relajado, porque escuchas lo que te gusta y no hay examen, llego a mi casa y con la información que te dan amplías conocimientos de lo que te gusta» (María José, 74 años, GD1).

Además, el mismo alumnado muestra su satisfacción hacia el profesorado por su labor docente, por la didáctica para la comprensión general de los contenidos programados.

"Hay una persona que es premio nacional de historia de este año, que nos está dando la asignatura de Europa, fue una conferencia magnifica y eso da siempre gusto escucharlo». (Maribel, 65 años, GD2).

«Fui military be aprendido en academias y demás, pero es distinto..., para mi es un orgullo que un catedrático nos imparta clases». (J. Carlos, 65 años, GD2).

Los contenidos académicos-científico de la UMEX son amplios y diversos, pues las materias son elegidas a demanda del alumnado. Las oportunidades de aprendizaje no se limitan únicamente a las clases, pues se ofrecen actividades extra-académicas que los participantes valoran mucho. Es otra forma de aprender a través del ocio creativo durante la vejez, que promueve la culturización del individuo, como ALV.

«Aqui hay otra cultura que es la que se coge visitando en viajes de estudios maravillosos». (Alicia, 58 años, GD2).

«Aparte de las clases, lo más enriquecedor son los talleres de canto, idiomas, arqueología, etc., y son todos maravillosos, pues lo mejor es que puedes hacer prácticas con ellos día a día» (Maximiliano, 63 años, GD3). 
"Cambia la forma de ver las cosas, pues hemos estado de excursión con la profesora de botánica y ahora puedo entender el significado..., habiendo disfrutando de lo que comprendes». (Ángela, 58 años, GD1).

\subsubsection{Valoración del profesorado}

La unanimidad del alumnado consultado valora positivamente la docencia que les permiten comprender con facilidad aquellos contenidos más complejos por la didáctica empleada en clase. Es clave que el profesorado de la UMEX sienta vocación por la enseñanza para «enganchar» a este público de edades avanzadas que asiste voluntaria y libremente, por el simple placer de aprender.

"Influye mucho el profesor, no basta con saber, hay que saber enseñary transmitir de modo ameno...» (Manolo, 62 años, GD2).

«Eso lo ves en la asistencia, cuando un profesor es bueno y te bace aprender, en sus clases se ve porque están siempre llenas...». (Emilio, 74 años, GD4).

«Hay clases que se hacen bastante largas, pero siempre aprendes algo nuevo gracias al profesor». (J. Antonio, 63 años, GD3).

«Destaco la clase de economia, jamás pensé que una clase de ese tema pudiera hacerse tan entretenida». (Ángela, 58 años, GD1).

\subsubsection{Democratización formativa}

Un aspecto destacado es el nivel académico del alumnado, pues se supuso al inicio de la investigación que predominarían personas sin acceso anterior a la enseñanza superior, cuando se constata que el alumnado está formado alrededor del 70\% por personas con nivel cultural medio-alto (Medicina, Derecho, Enfermería, Magisterio, etc.).

"Yo pondría que alrededor de un 15 o 20\% son personas que no ban estudiado nunca, los demás son personas que todos han estudiado y que tienen sus carreras». (Manolo, 62 años, GD2).

La oferta educativa de la UMEX está diseñada para que sea asequible a cualquier adulto mayor con independencia de niveles académicos. En los inicios de este programa universitario se realizaban pruebas de conocimientos básicos para la admisión, pero se eliminaron para favorecer el acceso universal y democrático de los adultos mayores.

\subsubsection{Mejoras de futuro}

Según indicó el alumnado consultado, dos puntos mejorables serían la falta de relación intergeneracional en los centros universitarios donde se imparten las clases, y las dificultades para la alfabetización digital.

Entre los objetivos programáticos de la UMEX es favorecer los encuentros intergeneracionales, siendo un propósito no logrado por la percepción del alumnado, ya que no tienen contactos apenas con los estudiantes de enseñanzas superiores regladas (Grados, postgrados,...), aún compartiendo espacios apropiados para el intercambio entre personas de distintas edades y generaciones.

La mayoría del alumnado manifiesta la escasa o nula relación con los universitarios jóvenes, sabiendo que sería beneficioso para estos grupos etarios. 
"En ese aspecto hay un vacio prácticamente total. No hay relaciones y no han propuesto ninguna actividad que permita comunicarnos con los jóvenes». (Teresa, 65 años, GD1).

«Eso aqui no se lleva a cabo, la primera relación que tengo con un universitario joven es contigo». (Manolo, 62 años, GD2).

«Sería bonito tanto para unos como para otros, porque los jóvenes serán mayores, y podrían preguntarnos muchas cosas sobre nuestras vidas, lo que hacemos y como lo afrontamos». (Manolo, 62 años, GD2).

«No hemos hecho ninguna actividad, lo poco que los ves en el descanso (en la cafetería de la Facultad), y ya està». (Clotilde, 81 años, GD1).

La UMEX está abierta para la matriculación a partir de los 55 años, y por tanto, se podrían considerar como relaciones intergeneracionales entre el propio alumnado cuando existen casi 30 años de diferencias entre los mismos. De cualquier modo, la UMEX es un espacio para la superación de la brecha generacional y el edadismo (Butler, 1975), contribuyendo así a la inclusión educativa sénior.

El otro punto mejorable es la integración de los adultos mayores en la sociedad de la información y del conocimiento, es decir, la alfabetización digital en el uso de las TIC. Este alumnado percibe como algunos tienen habilidades tecnológicas de usuarios, mientras que otros carecen de tales conocimientos necesarios hoy.

«Somos de la generación del lápizy la goma de borrar, y vendrían bien clases de informática pues tenemos dificultades, por ejemplo, con el móvilis. (Fernando, 61 años, GD3).

«Te dicen libros o sitios que visitar en Internet, pero no te enseñan cómo bacerlo...». (Rafael, 75 años, GD4).

«Me hace falta, pues tengo un déficit grande en el uso de las nuevas tecnologias, que es obligatorio en esta sociedad de Internet». (María José, 74 años, GD1).

\subsection{Perspectiva del profesorado}

El profesorado de la UMEX es académico en activo o jubilado, al igual que profesionales de distintos sectores productivos. Primeramente, señalan que el alumnado parece «tener hambre por aprender», de ahí las sensaciones de plenitud y satisfacción al impartir clases. El simple gesto de acabarlas con un aplauso del alumnado, es algo que en otros entornos de aprendizaje no han experimentado como valoración docente, según manifestaron unánimemente los tres profesores consultados.

«Se produce un efecto de interrelación, y eso pues te motiva (...) Ellos te dan mucho a ti, mucho, la satisfacción es enorme». (Fernando, profesor de Sociología).

"Fue realmente emocionante, nunca pensé que iba a sentir lo que siento cuando voy a la universidad de mayores (...) Desde el primer día para mi es la vida, porque las capacidades que tienen de atención, para preguntarte, (...) el interés es desmedido; ni en sueños hubiera tenido este auditorio». (Marisi, profesora de Literatura). 
"A mí es lo que me compensa de aqui es la satisfacción recibida. Una de las cosas que más te satisfacen es que puedas ser útil, entonces aqui te das cuenta de que hay mucho deseo de que cuentes y ves que muchos de ellos si siguieras media hora más estarían encantados». (Jacinto, profesor de Literatura).

«Espersonalmente, una necesidad de empatizar, con unas personas que veo que les gusta y que les estoy haciendo un bien» (Fernando, profesor de Sociología).

"Yo me siento tan querido que dices bueno (...), es mucho más lo que recibo que lo que yo pueda dar» (Jacinto, profesor de Literatura).

"Ya sabia un poco de lo que va la vida, pero siempre tengo que aprender más, y ellos siguen enseñándome por si me despisto. Rodearse de personas así siempre es constructivo». (Marisi, profesora de Literatura).

Este público sénior es respetuoso con el profesorado, y la peculiaridad es la asistencia mayoritaria a cada clase, sin esperar nada más a cambio que el conocimiento compartido.

«Desde el primer momento much a sintonia, much a complicidad, porque no hay ninguna fractura generacional...». (Jacinto, profesor de Literatura).

«La vida les ha tranquilizado, les ha enseñado (...) La tranquilidad en el sentido de que no hay estrés, no hay alteración hormonal, son personas reposadas que están en posición de valorar mucho más todo esto». (Jacinto, profesor de Literatura).

«El alumno universitario está abí, sus motivaciones, su desarrollo social, físico, biológico, tiene otras motivaciones que es generalmente aprobar (...) Este alumno va porque lo ha elegido». (Marisi, profesora de Literatura).

Sin duda, estas clases resultan de utilidad por sus contenidos académicos-científicos, además de otros elementos que enriquecen cultural y personalmente al alumnado.

"Los de sociología imprescindibles, porque lo primero que somos es eso: ser social, en eso nos distinguimos de los animales». (Fernando, profesor de Sociología).

"Creo que la literatura en general nos ayuda a vivir, a dar sentido a la existencia que a veces es complicado encontrarlo y más para este público que está en una etapa avanzada de su ciclo vital. La literatura llena mucha parte de la existencia, te nutre el sentido de la vida y esa es la intención que sigo en mis clases». (Marisi, profesora de Literatura).

Ese enriquecimiento personal se refleja en las relaciones intergeneracionales y las actitudes propositivas del alumnado, percibido en la alegría, la inquietud por saber y la participación social.

«La UMEX, se crea en toda España partiendo de un hecho, cuando le preguntabas a los mayores cuál era su máximo problema, siempre te decian: la soledad; más que la economía u otros problemas, la soledad». (Fernando, profesor de Sociología). 
"Aqui hay gente que les ha cambiado la vida, porque les permite socializar, tener un interés, se les abren las expectativas de la curiosidad...». (Jacinto, profesor de Literatura).

"Hay un aspecto muy importante de la universidad de mayores que es el social, el recreo es sagrado ya que es un momento social... se combina la formación cultural y la relación social y eso influye directamente en la prevención de todo». (Marisi, profesora de Literatura).

\section{Conclusiones}

Esta investigación confirma la hipótesis sobre la idoneidad del caso de estudio de la UMEX, como modelo pedagógico de práctica del ALV entre adultos mayores, por sus beneficios cognitivos y relacionales favorables al EA. Entendiendo así, la educación en la vejez como estrategia para la integración social y el desarrollo del talento sénior, es decir, la capacidad de elección de metas, la adecuada búsqueda de información, la gestión de las emociones, y el mantenimiento del esfuerzo necesario para alcanzar los objetivos (Marina, 2015).

El aprendizaje no sólo beneficia por la adquisición de conocimientos teóricos y prácticos, sino por la mejora cognitiva, emocional, fisiológica y funcional, en definitiva, la repercusión en la calidad de vida. Un adulto mayor que aprende, es un ciudadano preocupado de sí mismo y comprometido con los demás, que promueve el cambio social desde la experiencia de vida, pensando en su propia generación y en las venideras.

Los baby boomers demuestran capacidades de adaptación, aprendizaje y resiliencia, con niveles más alto de instrucción, nuevas demandas y necesidades formativas, además de nuevas formas de encarar la vida tras la jubilación. El dinamismo de la educación en la vejez irá articulando respuestas renovadas sobre el discurso educativo en relación al envejecimiento y la capacidad de aprendizaje en edades avanzadas (Serdio, 2015).

La UMEX y programas similares están desmitificando estereotipos y prácticas discriminatorias contra las personas mayores, la vejez y los procesos de envejecimiento. Quizás el reto de estos programas educativos sea la potenciación de entornos de solidaridad y aprendizaje intergeneracional para transitar de la brecha generacional hacia la huella generacional (Cambero, 2018).

En conclusión, se evidencia la importancia de la innovación en el aprendizaje para una vida longeva en comunidades educativas más democráticas, inclusivas y transformadoras, que permita la construcción de sociedades del conocimiento para todas las edades.

\section{Referencias bibliográficas}

Abellán García, Antonio; Ayala García, Alba; Pérez Díaz, Julio; Pujol Rodríguez, Rogelio (2018): “Un perfil de las personas mayores en España, 2018. Indicadores estadísticos básicos”. Informes envejecimiento en red, 17,34 .

Baigorri, Artemio y Chaves, Mar (2005): “The Digital Literacy of Older Citizens in Extremadura (Spain)” en Birgit Jæger (ed.): Young Technologies in Old Hands. An International View on Senior Citizen's Utilization of ICT. DJØF Publishing.

Bermejo García, Lourdes (2010). Envejecimiento activo y actividades socioeducativas con personas mayores: Guías de buenas prácticas. Madrid: Médica Panamericana. 
Butler, Robert. N. (1975). Why survive? Being old in America. Nueva York: Harper \& Row.

Cambero Rivero, Santiago (2015). Ciudadanía y voluntariado sénior en la sociedad telemática. Estudio de Avimex como paradigma del envejecimiento activo e inclusivo en Extremadura. Extremadura: Universidad de Extremadura.

Cambero Rivero, Santiago (2018): "De la brecha a la huella generacional". The Conversation. 19 de noviembre de 2018 (en línea). https://theconversation.com/de-la-brecha-a-la-huella-generacional-106953, consultado el día 19 de noviembre de 2018.

Chamahian, Aline (2005). Approche sociologique des Universités Tous Âges. Quel sens donner aux pratiques éducatives à l'heure de la retraite? Memoria de máster en investigación de sociología. Universidad de Provence.

Delors, Jacques (1996). La educación encierra un tesoro. (Informe a la UNESCO de la comisión internacional sobre la educación para el siglo XXI) (en línea). https://es.slideshare.net/marrisan/jacquesdelors-la-educacin-encierra-un-tesoro-informe-unesco, consultado el día 11 de septiembre de 2018.

ECC (2007). Join Report on Social Inclusion and Social Protection. Countries Profiles. Brussels: European Communities Council.

Fernández-Ballesteros, Rocío (2017). Psicología de la vejez: Una psicogerontología aplicada. Madrid: Pirámide.

Fernández-Ballesteros, Rocío (2000). Gerontología social. Madrid: Pirámide.

Giovanna Caprara, María (2017): "Promoción del envejecimiento activo" en Rocío Fernández-Ballesteros: Psicología de la vejez. Una psicogerontología aplicada. Madrid: Pirámide.

INE (2016). Encuesta sobre la participación adulta en las actividades de aprendizaje. Madrid: Instituto Nacional de Estadística.

INE (2017). Indicadores demográficos 2016. Madrid: Instituto Nacional de Estadística.

Lemieux, André (1987): “Gerontologie éducative et gérontagogie”. Lánnée gerontologique, 3, 213-219.

Marina, José Antonio (2015). Talento anciano: una pedagogía para la vejez (en línea). https://www.elconfidencial.com/alma-corazon-vida/educacion/2015-06-30/talento-anciano-una-pedagogia-parala-vejez-jose-antonio-marina_908839/, consultado el día 15 de diciembre de 2018.

Martínez Lozano, Isabel (2011). "Marco Europeo: 2012, Año europeo del envejecimiento activo y de la solidaridad intergeneracional" en IMSERSO, Libro Blanco del envejecimiento activo. Madrid: Ministerio de Sanidad, Política Social e Igualdad Secretaría General de Política Social y Consumo Instituto de Mayores y Servicios Sociales (IMSERSO).

Ministerio de Educación Cultura y Deporte (2015). Plan estratégico de aprendizaje a lo largo de la vida. Madrid: Ministerio de Educación Cultura y Deporte.

Montero, Isabel (2000): “Nuevas perspectivas en el aprendizaje de los mayores” en M Esther Alcalá y Valenzuela, Enrique (ed.): El aprendizaje de los mayores ante los retos del nuevo milenio. Madrid: Dykinson.

Naciones Unidas (2000). Seguimiento del Año Internacional de las Personas de Edad: Segunda Asamblea Mundial sobre el Envejecimiento. A/RES/54/262. 16 de junio. Nueva York: Naciones Unidas.

OMS (2002). Envejecimiento activo, un marco político. Madrid: Organización Mundial de la Salud. 
Pérez Díaz, Julio (2003): "La madurez de masas”. Imserso, Colección Observatorio de las personas Mayores, 12.

Roa Angarita, Claudia Janneth; Torres Puentes, Wilson (2014). “¿Comunidad educativa o sociedad educativa?". Educación y Ciudad, 27, 139-146.

Sáez Carreras, Juan (2003). Educación y Aprendizaje en las personas mayores. Madrid: SAFEKAT, S.L.

Sánchez Martínez, Mariano (2011). "Relaciones intergeneracionales” en IMSERSO: Libro Blanco del envejecimiento activo. Madrid: Ministerio de Sanidad, Política Social e Igualdad Secretaría General de Política Social y Consumo Instituto de Mayores y Servicios Sociales (IMSERSO).

Serdio Sánchez, Carmen (2015). "Educación y envejecimiento: Una relación dinámica y en constante transformación”. Educación XX1, 18 (2), 237-255.

UNESCO (2014). UNESCO Estrategia de educación 2014-2021. París: UNESCO.

Vellas, Pierre (1977). L'apport des Universités du Troisième Age en Abrégé de gérontologie. Masson.

Villar, Feliciano y Serrat, Rodrigo (2014): "La participación cívica como expresión de generatividad en la vejez". Estudos Interdisciplinares sobre o Envelhecimento, 19 (2), 345-358.

\section{Notas biográficas}

Santiago Cambero Rivero es Doctor en Sociología por la Universidad de Extremadura (UEX). Licenciado en CC. Políticas y Sociología, y en Derecho por la UNED. Máster en Gerontología Social por la Universidad de Barcelona. Profesor Asociado del Departamento de Dirección de Empresas y Sociología (UEX), impartiendo clases en la Facultad de Educación en los Grados de Educación Infantil y Primaria. Miembro del grupo de investigación «Análisis de la Realidad Social» (UEX). Socio de la Federación Española de Sociología (FES), European Network in Aging Studies (ENAS) y Colegio de Sociología y Ciencias Políticas de Extremadura. Líneas de investigación: Sociología de la Vejez y Sociología de la Educación.

Deborah Díaz Galván es Graduada en Educación Infantil por la Universidad de Extremadura (UEX). Líneas de investigación: Sociología de la Educación y Sociología de las Edades y Ciclo de Vida. 